\title{
Flexible and Conductive Cellulose Substrate by Layered Growth of Silver Nanowires and Indium-doped Tin Oxide
}

Tao Tao, ${ }^{\text {a }} \mathrm{Xi}$ Liu, ${ }^{\mathrm{a}}$ Amjad Islam, ${ }^{\mathrm{a}}$ Junying Wu, ${ }^{\mathrm{a}}$ Yonghao Ni, ${ }^{\mathrm{a}, \mathrm{b}}$ Liulian Huang, ${ }^{\mathrm{a}}$

Lihui Chen, ${ }^{a}$ Xinhua Ouyang, ${ }^{a}$ and Jianguo $\mathrm{Li}^{\mathrm{a}}{ }^{\mathrm{a}}$,

\begin{abstract}
Regenerated cellulose film (RCF) has potential as a conductive substrate due to features such as its degradability, transparency, and flexibility. Indium doped tin oxide (ITO) is a conventional conductive material, but its rigidity restricts the formation of flexible conductive film. In this study, silver nanowires (AgNWs) were introduced between the RCF and the ITO conductive framework. Additionally, the fabrication of flexible, conductive, and transparent RCF was conducted. The AgNWs-ITO based RCF demonstrated high conductivity ( $170 \Omega$ per sq) and transparency $(78 \%)$ by the addition of $50 \mu \mathrm{L}$ of AgNWs. After bending the sample 50 times with a $5 \mathrm{~mm}$ curve radius, the as-prepared conductive RCF presented an electric resistance improvement of $19 \%$, with a $485 \%$ increase for the control ITObased RCF. This is a result of the AgNWs framework, which can lessen the destruction of the bending treatment on the conductive layer and can also desirably connect the ITO conductive sections. The novel approach can expedite the versatile applications of flexibly conductive RCF on printable, portable, and wearable electronic devices.
\end{abstract}

Keywords: Conductive regenerated cellulose film; Indium doped tin oxide; Silver nanowires; Flexibility

Contact information: a: College of Material Engineering, Fujian Agriculture and Forestry University, Fuzhou 350002, China; b: Limerick Pulp and Paper Centre, Department of Chemical Engineering, University of New Brunswick, Fredericton, New Brunswick E3B 5A3, Canada;

* Corresponding author: Jianguo Li, E-mail: jianguolicn@fafu.edu.cn

\section{INTRODUCTION}

As one of the most abundant natural resources, cellulose, is characterized by unique features including renewability, biodegradability, low thermal expansion, and eco-friendly properties (Yan and Xu 2015; Liu et al. 2017; Zhang et al. 2018, 2019a). This could lead to the development of light weight and low-cost green products. One of these productscellulose film - is versatile and has a facile chemical modification, smooth surface, controllable film structure, and transparency (Wu et al. 2009, 2020; Huang et al. 2019; Zheng et al. 2019). Furthermore, in terms of functionality, the regenerated cellulose film (RCF) can be optimized from the level of the cellulose molecule, which can prepare tunable and delicate cellulose-based products.

RCF has been employed in several applications (Li et al. 2014, 2018; Wu et al. 2009). For example, RCF has been employed in the antibacterial packing material, adsorption and catalytic carrier, filter membrane, and even the conductive substrate for photoelectric devices. The conductive and transparent RCF can be supportive in the manufacturing of flat-panel displays, wearable electric devices and sensors, defrosting windows, etc. (Cao et al. 2014; Lee et al. 2014; Zhang et al. 2019b). More importantly, transparent RCF presents superior flexibility; thus it could be favorable in the foldable and light-weight electronic devices. Compared with its counterparts, such as glass (Li et al. 
2018) and plastic (polycarbonate and polyethylene terephthalate) (Lee et al. 2014), RCF is flexible, light weight, and eco-friendly.

To prepare conductive RCF, conductive material is introduced onto the surface of $\mathrm{RCF}$, which is required because pure RCF is an insulator. The conductive materials for decorating RCF mainly consist of metals and metal nanowire, carbon-based materials, conductive polymers, and doped metal oxide (Liu et al. 2014; Tang et al. 2014; Lee and Jeong 2015; Raghunathan et al. 2017; He et al. 2018; Zhang et al. 2019c). Both metals and metal nanowire present excellent ductility and conductivity for the flexible substrate. However, the low transparency (metal luster) for the thin layer in metal, high surfaceroughness, and the poor adhesion between the metal nanowire and substrate limit their performance as well as their commercial applications. Carbon-based materials have high transparency and flexibility but possess low electrical conductivity. The carbon-based materials also contain complicated synthesis processes accompanied by environmental issues. The conductive polymer can have a high conductivity with a reduced transparency, especially in the visible wavelength region. Indium-doped tin oxide (ITO) is characterized by excellent optical and electronic properties with high environmental stability. However, due to its brittle nature, ITO has poor compatibility with flexible substrates and is difficult to use in high-throughput roll-to-roll processing. This results in a challenging and urgent problem for constructing the flexible and transparent ITO-based device.

In this paper, ITO and $\mathrm{Ag}$ nanowires (AgNWs) were employed as the integrated conductive layer on the RCF surface to prepare flexible, transparent, and conductive RCF. In this conductive layer, the AgNWs formed the conductive framework, and ITO was padded into the AgNWs framework. The advantages of conductivity, flexibility, and transparency were achieved simultaneously through the novel configuration of this conductive layer. The developed technology provides a great potential to accelerate the cellulose diversified applications and high value-added products.

\section{EXPERIMENTAL}

\section{Materials}

The regenerated cellulose film (RCF) was prepared by the dissolution of softwood dissolving pulp in an $85 \% \mathrm{~N}$-methylmorpholine-N-oxide (NMMO) aqueous solution. It was regenerated in deionized water. The RCF was $10 \mu \mathrm{m}$ thick and had a transparency of $93 \%$. Indium tin oxide (ITO) having a target diameter equal to $50 \mathrm{~mm}\left(\mathrm{SnO}_{2}\right.$ to $\mathrm{In}_{2} \mathrm{O}_{3}$ equal to 10 to 90) was obtained from Zhongnuoxincai Corporation (Beijing, China). A $0.5 \%$ AgNWs-isopropanol suspension was purchased from Nanjing XFNANO Materials TECH Co., Ltd. (Nanjing, China), where the diameter of AgNWs was 100 to $200 \mathrm{~nm}$ and the length was 30 to $50 \mu \mathrm{m}$.

\section{Preparation of ITO-based Conductive RCF}

The ITO-based conductive RCF was prepared by sputtering ITO on the regenerated cellulose film (RCF). The sputtering treatment was carried out from a magnetron sputtering system (TRP-450, Shenyangkexueyiqi Corporation, Shenyang, China), based on a reference procedure (Li et al. 2018). First, the RCF and ITO were fixed on the sample table and sputtering table, respectively. There was a spatial distance of $30 \mathrm{~cm}$ between them. Subsequently, the vacuum was increased to $6 \times 10^{-4} \mathrm{~Pa}$ by using a combined mechanical pump and molecular pump. The working pressure was adjusted to 3.0 $\mathrm{Pa}$ with an Ar flow 
of $30 \mathrm{sccm}$ at $90{ }^{\circ} \mathrm{C}$. A direct current was employed for sputtering the ITO on the RCF at $120 \mathrm{~W}$ power density for a specific time. After the sputtering treatment, the ITO-RCF was held in a zip lock bag in a nitrogen atmosphere.

\section{Preparation of AgNWs Promoted ITO-RCF}

The AgNWs was inserted between the ITO and RCF through the spin-coating method. Firstly, diluted AgNWs solutions with different concentrations were prepared by adding the targeted volumes of the original AgNWs solution $(30 \mu \mathrm{L}, 50 \mu \mathrm{L}, 70 \mu \mathrm{L}$, and $100 \mu \mathrm{L}$ ) into isopropanol (total volume was $200 \mu \mathrm{L}$ ). A 3 by $3 \mathrm{~cm}^{2} \mathrm{RCF}$ was cast on the spin coater (KW-4A, Institute of Microelectronics of the Chinese Academy of Sciences, Hefei, China) with a dropping of $200 \mu \mathrm{L}$ diluted AgNWs solution. This was performed with the condition of $1000 \mathrm{rpm}$ in $30 \mathrm{~s}$. The AgNWs-based RCF was dried at room temperature for the subsequent analyses. The ITO was sputtering onto the AgNWs-RCF by following the same procedures described above.

\section{Characterization}

The electrical conductivity was determined by using a four-point resistance tester (KDB-3, KunDe Inc., China). The transparency of samples was detected by employing a UV-vis spectrophotometer (Agilent UV-8453, Beijing, China). The morphologies were investigated using a scanning electron microscope (SEM, Hitachi SU8010, Schaumburg, China). The bending resistance of the conductive RCF was indicated by the conductive resistance change. This was indicated after the bending treatment that had a $5 \mathrm{~mm}$ radius. The conductive resistance change was calculated according to Eq. 1,

$$
\text { Conductive resistance change }(\%)=\left(R_{1}-R_{0}\right) \div R_{0} \times 100 \%
$$

where the $R_{0}$ and $R_{l}$ were the conductive resistances ( $\Omega$ per sq) before and after the bending treatment. The flexible performance of the device was tested by using a multichannel physiological signal acquisition and process system (RM6240CD, Chengdu Instrument Factory, China). Two pieces of conductive RCFs were separated by a spacer (a sponge was used to prevent the direct connection of the two RCFs). They were fixed onto the device and encapsulated with copper straps.

\section{RESULTS AND DISCUSSION}

\section{Properties of ITO-based Conductive RCF}

Figure 1 shows the effect of the sputtering ITO treatment on the conductivity and transparency of the conductive RCF. Pure RCF is an insulator, while the conductivity of RCF can be remarkably improved by the sputtering treatment of ITO. Increasing the treatment time can considerably decrease the conductive resistance of the RCF sample (from Fig. 1). For example, an ITO sputtering in $0.5 \mathrm{~h}$ prepared the conductive RCF with $450 \Omega$ per sq from the pure RCF with infinite resistance. By increasing the sputtering time from 0.5 to $1.5 \mathrm{~h}$, the conductive resistance was decreased to $139 \Omega$ per sq and even reduced to $41 \Omega$ per sq after $2.5 \mathrm{~h}$ of ITO sputtering. On the other hand, the conductive resistance of RCF was characterized by a non-linear decrease by increasing the sputtering time. From Fig. 1, the resistance decreased rapidly in the beginning, but this reduction occurred slowly in the second phase (even reaching a plateau). This result agreed with the previous reports (Carvalho et al. 2005). The effect of ITO sputtering on the transparency at $550 \mathrm{~nm}$ 
wavelength of RCF is shown in Fig. 1. The ITO sputtering treatment slightly decreased the $\mathrm{RCF}$ transparency. This is where a simple linear relation between the RCF transparency and ITO sputtering time was presented during the sputtering process. This can be ascribed to the prolonged sputtering treatment, which resulted in a larger amount and thickness of ITO on the RCF surface. This ultimately reduces the transparency of the conductive RCF. Sahu et al. (2018) found that the thickness of the ITO layer affected the conductivity and transparency. In Sahu et al. (2018), the resistivity decreased from $1.3 \times 10^{-3} \Omega \mathrm{cm}$, to $6.5 \times$ $10^{-4} \Omega \mathrm{cm}$, and finally to $5.8 \times 10^{-4} \Omega \mathrm{cm}$. The transparency changed from $82 \%$, to $76 \%$, and finally to $71 \%$. This occurred when the ITO thickness increased from $20 \mathrm{~nm}$, to $30 \mathrm{~nm}$, and finally to $60 \mathrm{~nm}$.

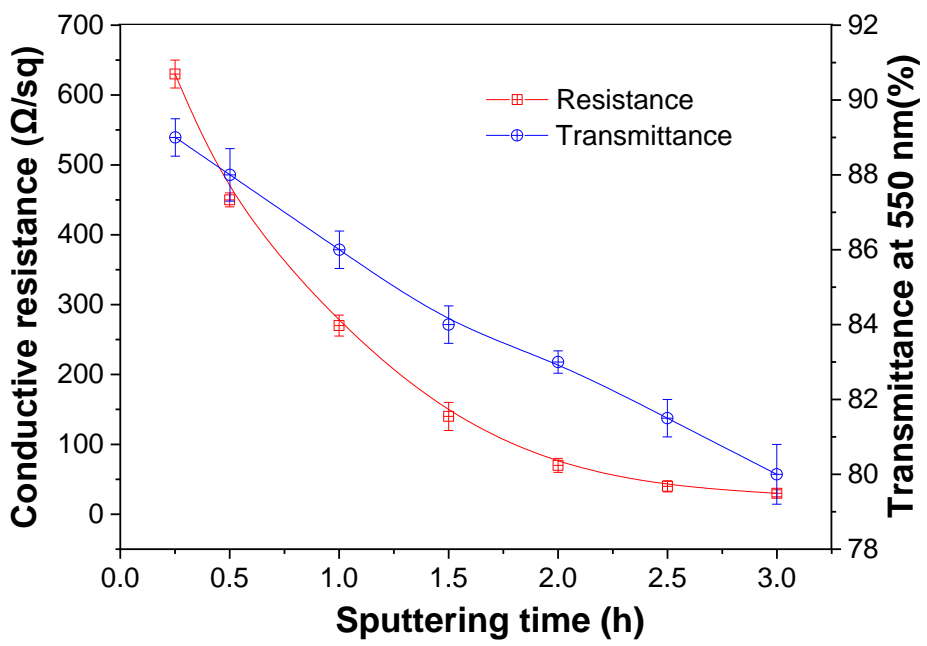

Fig. 1. Conductive RCF as a function of the ITO sputtering treatment

\section{AgNWs Promoted the Properties of ITO-based Conductive RCF}

To harvest at a high conductivity/low resistance and high transparency, a $1 \mathrm{~h}$ treatment time was the optimized condition for the ITO-RCF (Fig. 1). The subsequent analyses were performed on samples obtained under this condition for $270 \Omega$ per sq of conductive resistance and $86 \%$ transparency. In order to further modify the ITO-RCF properties, the AgNWs framework was introduced as the buffer layer between the RCF substrate and the ITO conductive layer. Owing to the excellent electrical conductivity of $\mathrm{Ag}$, a small amount of AgNWs was employed, thus presenting an AgNWs conductive framework on the RCF surface. As shown in Fig. 2, the AgNWs buffer framework considerably improved the conductivity and decreased the sheet resistance of the conductive RCF. An amount of $30 \mu \mathrm{L}$ of AgNWs decreased the conductive resistance to $210 \Omega$ per sq from $270 \Omega$ per sq of the original ITO-RCF. The resistance was reduced to $170 \Omega$ per sq and $120 \Omega$ per sq from $50 \mu \mathrm{L}$ and $100 \mu \mathrm{L}$ of AgNWs, respectively.

Figure 3 shows the effect of AgNWs framework on the transparency of the ITObased conductive RCF. AgNWs had a negative impact on the transparency of the conductive RCF. When the amount of AgNWs was $50 \mu \mathrm{L}$ and $100 \mu \mathrm{L}$, the transparency of the conductive RCF was $78 \%$ and $74 \%$, which was much lower than that of the control conductive ITO-RCF (86\%, Fig. 3). Compared with the transparency of conductive ITObased RCF, the AgNWs showed a more negative effect than the single ITO. For the $140 \Omega$ per sq resistance of the conductive RCF, the transparency was still $84 \%$ for the ITO-RCF (Fig. 1) and only $76 \%$ of the ITO-AgNWs-RCF (Fig. 2 and 3). Almost $81 \%$ of the transparency for the conductive RCF was followed by $40 \Omega$ per sq resistance of the ITO- 
RCF (Fig. 1) and $210 \Omega$ per sq of the ITO-AgNWs-RCF (Fig. 2 and 3). A larger reduction occurred in the transparency of the conductive RCF due to the AgNWs framework when compared to the single ITO. This can be attributed to the metallic luster of AgNWs (Cao et al. 2014), whereas the ITO possesses a relatively high transparency because of its wide band gap. In this studied condition, the AgNWs-based conductive RCF with a $50 \mu \mathrm{L}$ dosage of AgNWs exhibited an $88 \%$ transmittance with a $1200 \Omega$ per sq conductivity. Mun et al. (2016) prepared the strain sensor of AgNWs on cellulose; AgNWs resulted in a low transparency. The transparency was just $70 \%$ when the sheet resistance was $2.4 \mathrm{k} \Omega$ per sq at $0.01 \mathrm{wt} \%$ of AgNWs.

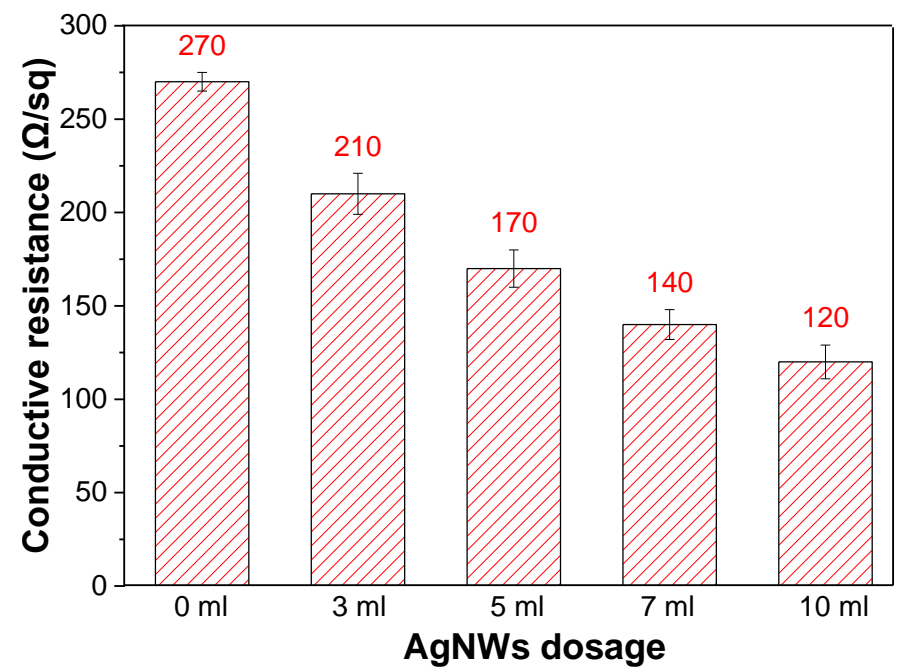

Fig. 2. The effect of AgNWs on the conductivity of the ITO-based conductive RCF

a)

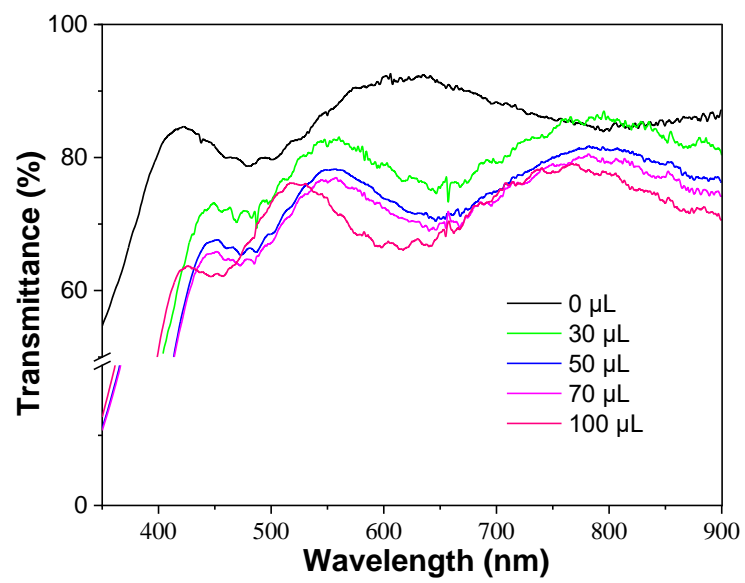

b)

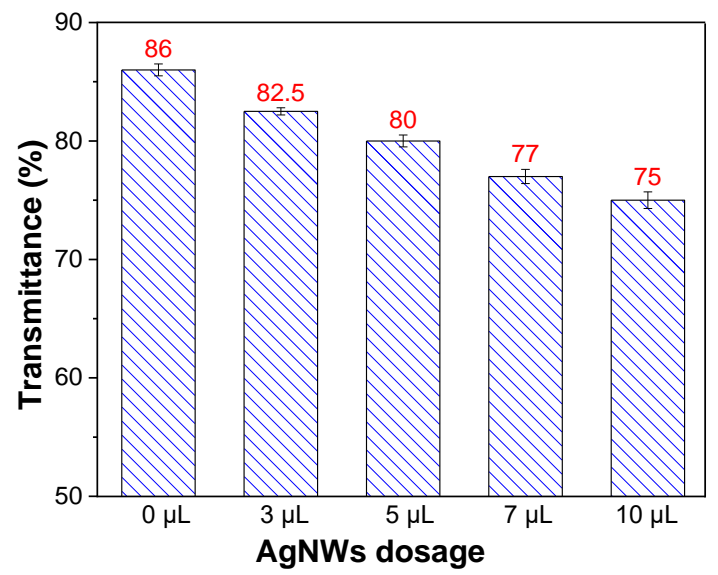

Fig. 3. The effect of AgNWs on the transparency of the ITO-based conductive RCF 
Figure 4 presents the SEM observation of the conductive layers on the RCF surface. From Fig. 4a), the ITO layer showed a smooth surface with a roughness of $15 \mathrm{~nm}$, which was located on the RCF. The AgNWs formed a conductive framework due to the small amount and loose deposit on the RCF surface (shown in Fig. 4c) and d). Based on the approach of spin-coating AgNWs followed by depositing ITO on the $\mathrm{RCF}$, an integrated conductive layer clearly formed with the AgNWs framework and ITO filler. It was a desirable configuration for the flexible, transparent, and conductive RCF with low roughness of $30 \mathrm{~nm}$, which will be discussed in the next section.
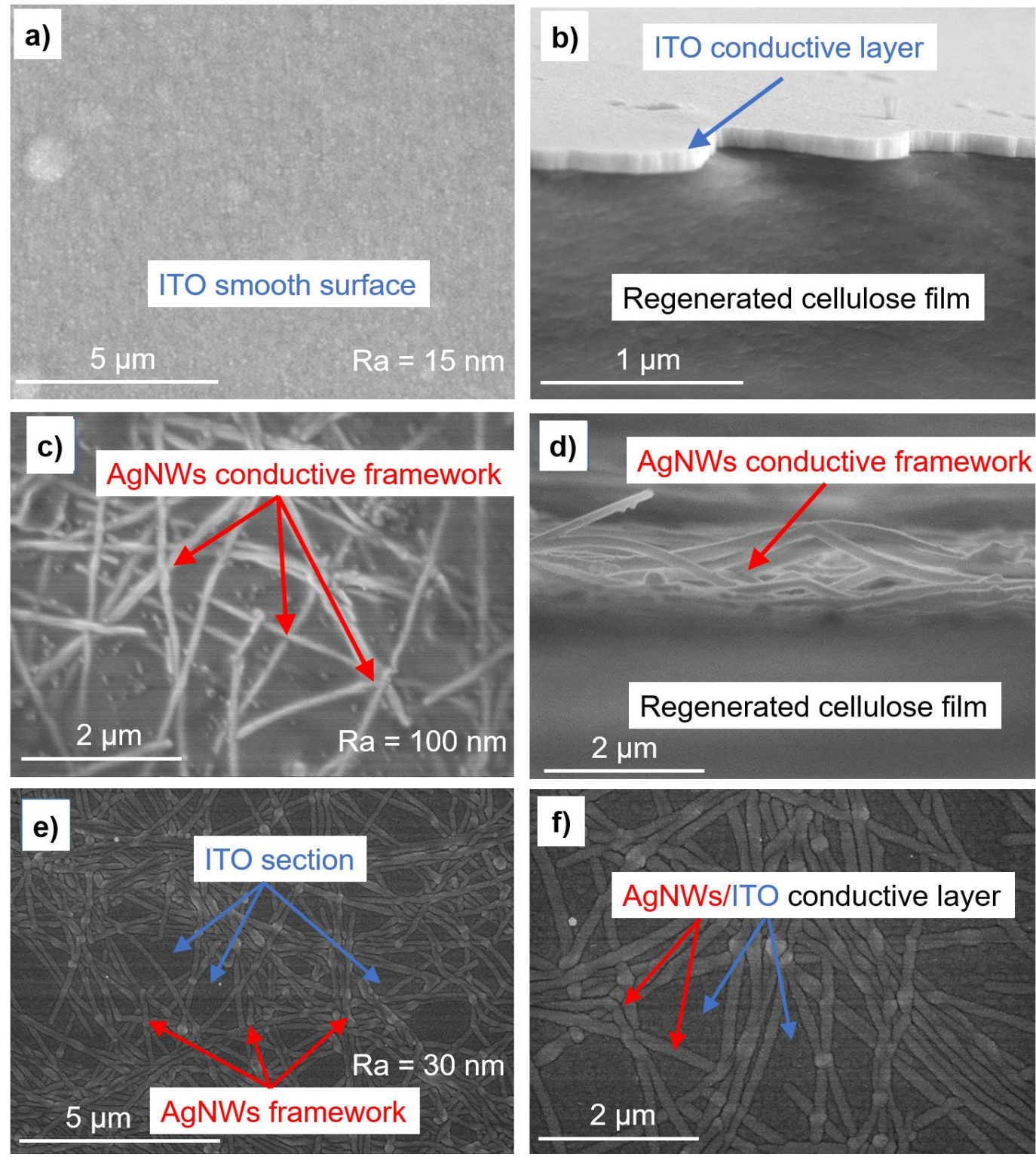

Fig. 4. Morphologies of the conductive RCF: a) ITO surface, b) ITO cross-section, c) $50 \mu \mathrm{L}$ AgNWs surface, d) $50 \mu \mathrm{L}$ AgNWs cross-section, e) and f) surface of $50 \mu \mathrm{L}$ AgNWs framework and ITO filler. $R_{a}$ : roughness of conductive RCF. 
Figure 5 shows the effect of the bending treatment on the resistance of transparent and conductive RCF. The ITO-based conductive RCF without AgNWs had an inferior bending resistance and five times of the bending treatment increased the conductive resistance from $270 \Omega$ per sq to $452 \Omega$ per sq. The resistance was further increased to 1580 $\Omega$ per sq by bending 50 times. This indicated the remarkable decrease of conductivity of the ITO-based conductive RCF due to the bending treatment. As the inorganic conductive layer, the ITO was characterized by high rigidity, friableness, and low flexibility (Lee et al. 2014; Tran et al. 2015). The ITO layer was fractured by the bending treatment, thus disturbing the current carrier mobility. It finally considerably decreased the conductivity of the ITO-based conductive RCF. The results agreed with those reported in the literature that the ITO-based conductive substrates were not suitable for developing flexible conductive devices (Cao et al. 2014; Li et al. 2018).

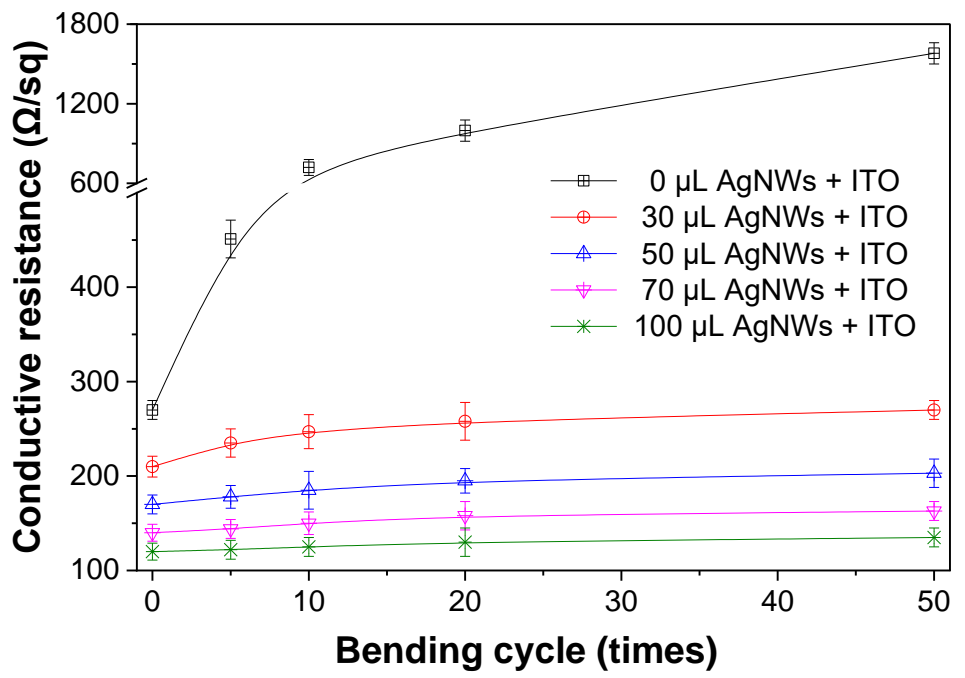

Fig. 5. The effect of AgNWs framework on the bending capacity of conductive ITO-RCF

AgNWs present outstanding toughness and flexibility (Cao et al. 2014; Lee et al. 2014). These features can be favorable for the bending resistance of ITO-based conductive RCF. Figure 5 presents that with the addition of $30 \mu \mathrm{L} \mathrm{AgNWs,} 5$ times of bending treatment only increased the conductive resistance from $210 \Omega$ per sq to $235 \Omega$ per sq (increased $25 \Omega$ per sq, much lower than $182 \Omega$ per sq of single ITO-RCF, from $270 \Omega$ per sq to $452 \Omega$ per sq). 50 times of bending resulted in an increase of $60 \Omega$ per sq from $210 \Omega$ per sq, to $270 \Omega$ per sq. The bending resistance of the conductive RCF can be further modified due to increasing the amount of $\mathrm{AgNWs}$. In relation to the bending treatment of 50 times, the resistance change of the conductive RCF was $33 \Omega$ per sq for $50 \mu \mathrm{L} \mathrm{AgNWs}$ (from $170 \Omega$ per sq to $203 \Omega$ per sq), while only $15 \Omega$ per sq for $100 \mu \mathrm{L}$ of $\mathrm{AgNW}$ (from $120 \Omega$ per sq to $135 \Omega$ per sq), respectively. One study (Hu et al. 2010) has demonstrated less of an increase in sheet resistance for the AgNWs than in ITO by the bending treatment. The results went from $17 \Omega$ per sq to $21 \Omega$ per sq and from $51 \Omega$ per sq to $6 \mathrm{k} \Omega$ per sq after 100 times of bending in $5 \mathrm{~mm}$. Sannicolo et al. (2016) also elucidated that the AgNWs presented a more negligible increase in electrical resistance for bending and better responses of stretching than the ITO. The AgNWs was favorable for manufacturing flexible electrodes. 

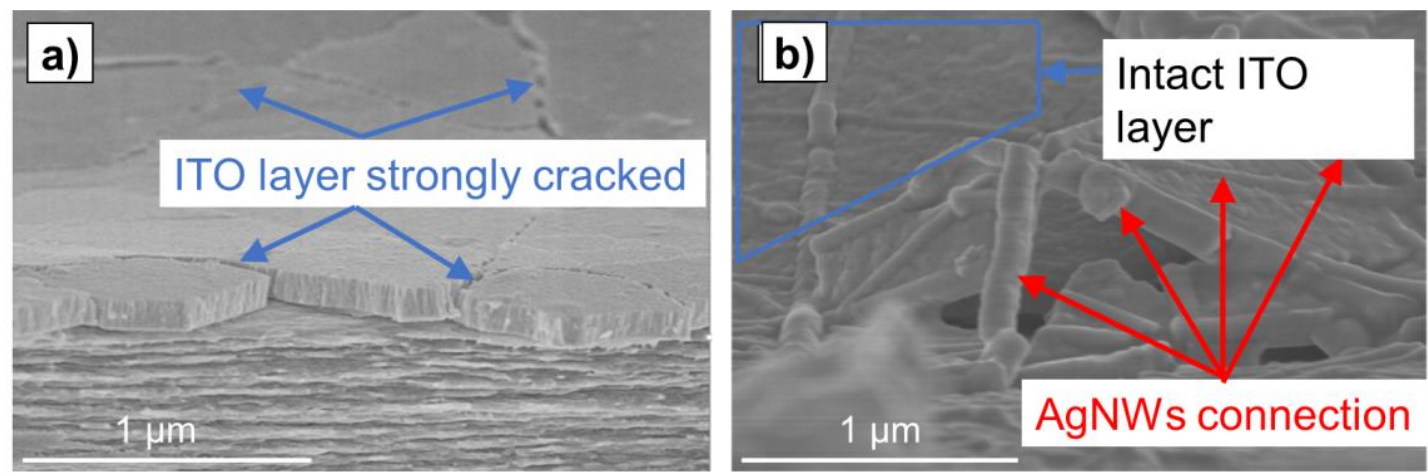

Fig. 6. Morphologies of the conductive RCF after the bending treatment: a) ITO layer, b) $50 \mu \mathrm{L}$ AgNWs framework and ITO filler

The effect of the bending treatment on the conductive RCF morphology is presented in Fig. 6. Due to the fragile nature of ITO, the bending treatment remarkably destroyed and cracked the ITO conductive layer. The bending treatment even led to the peeling of the ITO from the RCF (Fig. 6a). The resultant fracture of the ITO layer hindered the electron transportation, thus considerably decreased the conductivity of ITO-based conductive RCF (largely increasing its conductive resistance in Fig. 5) due to the bending treatment. As shown in Fig. 6b, the bending treatment presented a weaker effect on destroying and deconstructing the ITO layer for the AgNWs-ITO sample than that of the ITO's single sample. This was because the AgNWs can absorb and transfer the bending stress. As a result, it was desirable to protect the fragile ITO conductive layer. Moreover, although the integrated AgNWs-ITO conductive layer was partial destroyed, the flexible AgNWs were favorable for linking and connecting. The AgNWs framework was also supportive for the electron transportation in the integrated conductive substrate. Based on this, the AgNWs-ITO conductive RCF presented a lower loss of conductivity than the control ITO based RCF after the bending treatment.

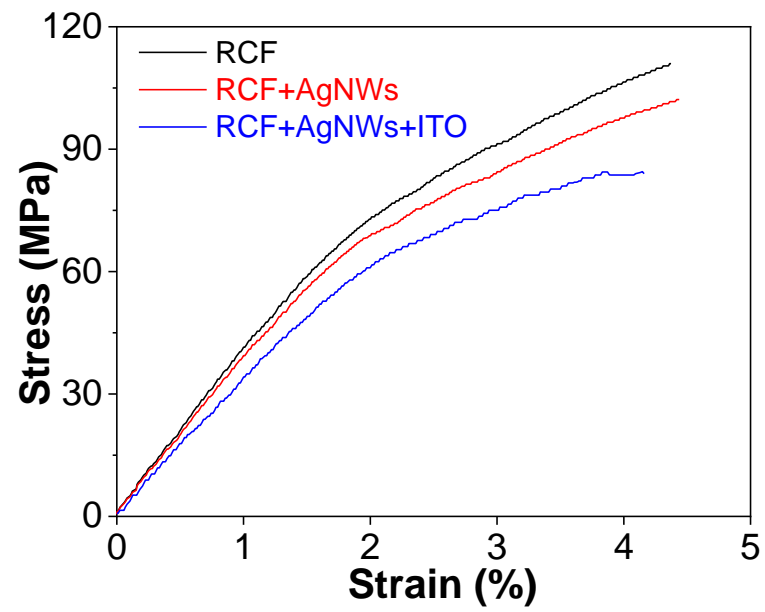

Fig. 7. The strength of the conductive RCF. $50 \mu \mathrm{L}$ AgNWs surface; $1 \mathrm{~h}$ sputtering time of ITO 
The mechanical strength of conductive regenerated cellulose film with AgNWs and ITO was investigated (Fig. 7). The original RCF showed a tensile strength of about 110 $\mathrm{MPa}$ with a strain at break of $4.4 \%$. The conductive RCF with AgNWs presented a similar stress-strain curve with the original RCF. When introducing ITO onto the RCF, the strength of conductive regenerated cellulose film was slightly weaker than the original cellulose film. This may due to the fact that ITO can insert itself into the inner structure of cellulose film, thus disturbing the bonding of cellulose molecules.

\section{Highly Flexible Devices Based on Conductive Regenerated Cellulose Film}

The flexible and conductive RCF with AgNWs interface modification was employed for constructing a simple electronic device. The high flexibility of the RCFbased device was further demonstrated by investigating the effect of the bending treatment on the conductivity performance of the devices. When there was no pressure on the device, the electric circuit was in the off state with the indication of enough high resistance, as shown in Fig. 8a). Bending the device can achieve the connection of the conductive substrates; thus a superior electric circuit was built (Fig. 8b). Figure 8 further describes the flexibility performance of the electric device.
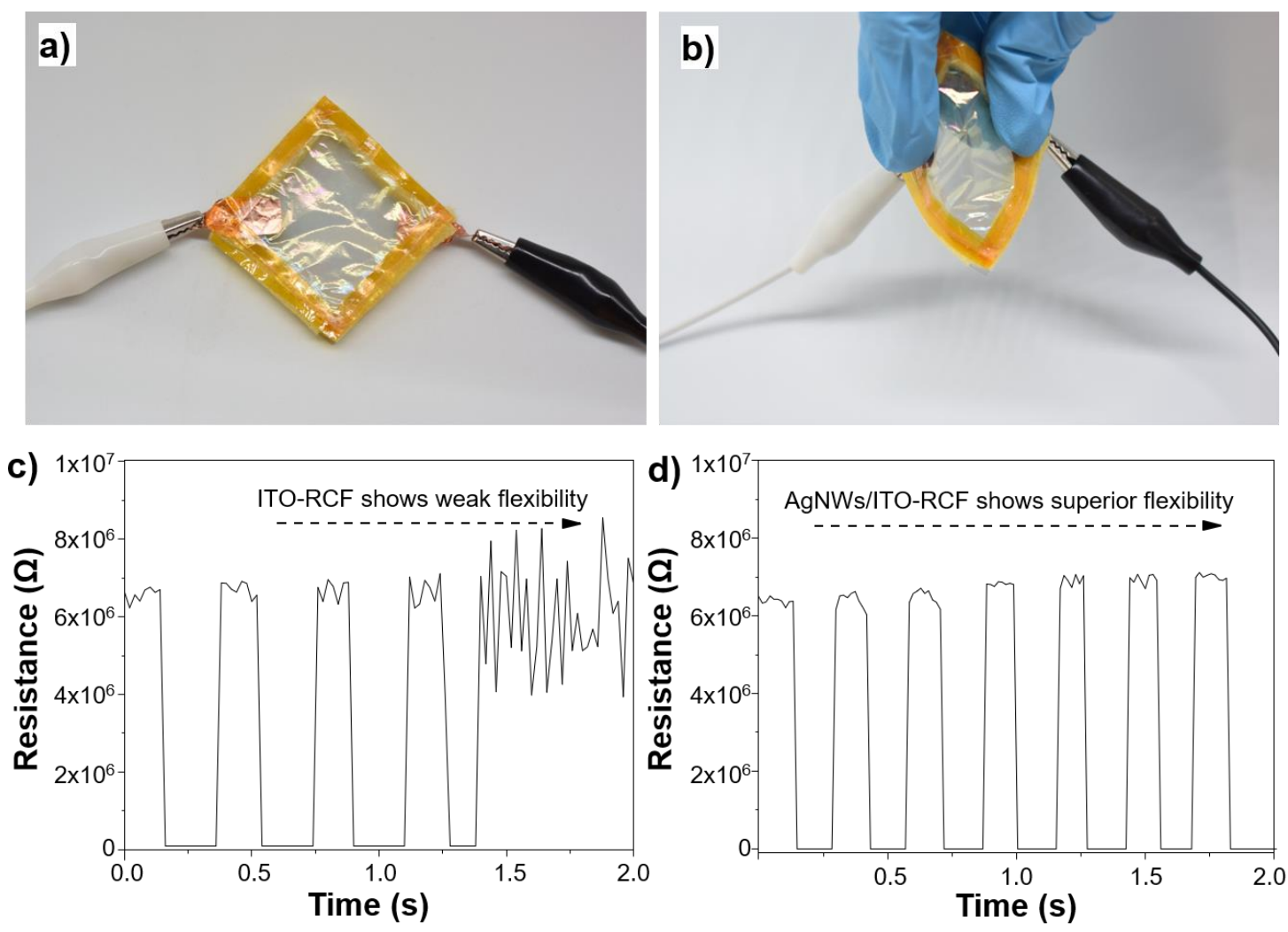

Fig. 8. Flexibly electric device: a) Commonly, the electric device was in the off state with the indication of sufficiently high resistance due to the non-connection of the two RCFs, b) Bending the device can achieve the connection of the conductive substrates, thus build an electric circuit, $\mathrm{C}$ ) The control ITO-RCF showed weak performance relative to bending, due to the conductivity decrease after bending, d) AgNWs modified ITO-RCF illustrates an outstanding flexibility, where a regular change of conductive resistance appears when bending and releasing the device 
The control ITO-RCF based device illustrated a strong decrease in the conductive performance and even exhibited non-conductivity after several bending treatments. This was a response to the weak flexibility of this kind of device due to the rigid and brittle feature of the ITO (Fig. 8c). However, the AgNWs interface modification endowed the RCF-based electric device with high flexibility and toughness. This feature was indicated by the regular change of resistance, thus was in an insulated state when no connection existed in the conductive RCF. The conductive RCF was in a conductive state of $250 \Omega$ during bending (Fig. 8d).

\section{CONCLUSIONS}

1. Conductive RFC was developed with the superior features of the high conductivity (170 $\Omega$ per sq) and transparency (78\%). This was accomplished by the layered growth of spin-coating the AgNWs and sputtering the ITO.

2. Based on the cooperation of AgNWs and ITO, the conductive RCF showed desirable flexibility, where the conductivity was much lower than the control conductive RCF after the bending treatment.

3. The conductive, transparent, and flexible RCF was successfully used to construct a flexible device with a high sensitivity and tolerance.

\section{ACKNOWLEDGMENTS}

This work was financially supported by the National Natural Science Foundation of China (Grant No. 31700520, No.21674123, No. 31971612), the Natural Science Foundation of Fujian Province (Grant No. 2018J05040), the Distinguished Young Scholars of Fujian Agriculture and Forestry University (Grant No. xjq201729), Scientific and technological innovation funding of Fujian Agriculture and Forestry University (Grant No. CXZX2017040, CXZX2018007), and the China Scholarship Council (201808350033).

\section{REFERENCES CITED}

Cao, W., Li, J., Chen, H., and Xue, J. (2014). “Transparent electrodes for organic optoelectronic devices: A review," J. Photon. Energy 4(1), 1194-1198. DOI: 10.1117/1.JPE.4.040990

Carvalho, C. N., Lavareda, G., Fortunato, E., Alves, H., Gonçalves, A., Varela, J., Nascimento, R., and Amaral, A. (2005). "ITO films with enhanced electrical properties deposited on unheated ZnO-coated polymer substrates," Mat. Sci. Eng. $B$ 118(1-3), 66-69. DOI: 10.1016/j.mseb.2004.12.015

He, S., Xin, B., Chen, Z., and Liu, Y. (2018). "Flexible and highly conductive Ag/Gcoated cotton fabric based on graphene dipping and silver magnetron sputtering," Cellulose 25(6), 3691-3701. DOI: 10.1007/s10570-018-1821-4

Hu, L., Kim, H. S., Lee, J. Y., Peumans, P., and Cui, Y. (2010). "Scalable coating and properties of transparent, flexible, silver nanowire electrodes," ACS Nano 4(5), 2955 2963. DOI: $10.1021 / \mathrm{nn} 1005232$ 
Huang, J., Liu, Y., Sun, B., Li, J., Zhang, R., and Nie, S. (2019). "Laccase pretreatment for enhancing microwave-assisted alkaline extraction of hemicellulose from bagasse," BioResources 14(1), 931-942. DOI: 10.15376/biores.14.1.931-942

Lee, J., Woo, J. Y., Kim, J. T., Lee, B. Y., and Han, C. S. (2014). "Synergistically enhanced stability of highly flexible silver nanowire/carbon nanotube hybrid transparent electrodes by plasmonic welding," ACS Appl. Mater. Inter. 6(14), 1097410980. DOI: 10.1021/am502639n

Lee, T. W., and Jeong, Y. G. (2015). "Regenerated cellulose/multiwalled carbon nanotube composite films with efficient electric heating performance," Carbohyd. Polym. 133, 456-463. DOI: 10.1016/j.carbpol.2015.06.053

Li, J., Yang, H., Huang, K., Cao, S., Ni, Y., Huang, L., Chen, L., and Ouyang, X. (2018). "Conductive regenerated cellulose film as counter electrode for efficient dyesensitized solar cells," Cellulose 25(9), 5113-5122. DOI: 10.1007/s10570-018-1913-1

Li, J., Liu, X., Zheng, Q., Chen, L., Huang, L., Ni, Y., and Ouyang, X. (2019). "Urea/ $\mathrm{NaOH}$ system for enhancing the removal of hemicellulose from cellulosic fibers," Cellulose 26(11), 6393-6400. DOI: 10.1007/s10570-019-02587-7

Li, S., Liu, S., Huang, F., Lin, S., Zhang, H., Cao, S., Chen, L., He, Z., Ryan, L., Yang, J., Ni, Y., and Huang, L. (2018). "Preparation and characterization of cellulose-based nanofiltration membranes by interfacial polymerization with piperazine and trimesoyl chloride," ACS Appl. Mater. Inter. 6(10), 13168-13176.

DOI: 10.1021/acssuschemeng.8b02720

Liu, K., He, B., Qian, L., and Li, J. (2014). “Conducting graphite/cellulose composite film as a candidate for chemical vapor-sensing material," BioResources 9(3), 52795289. DOI: 10.15376/biores.9.3.5279-5289

Liu, X., Jiang, Y., Xie, Q., Nie, S., and Song, X. (2017). "Effect of alkali pectinase pretreatment on bagasse soda-anthraquinone pulp," BioResources 12(3), 5045-5056. DOI: 10.15376/biores.12.3.5045-5056

Mun, S., Zhai, L., Min, S. K., Yun, Y., and Kim, J. (2016). "Flexible and transparent strain sensor made with silver nanowire-coated cellulose," J. Intel. Mat. Syst. Str. 27(8), 1011-1018. DOI: 10.1177/1045389X15577651

Raghunathan, S. P., Narayanan, S., Poulose, A. C., and Joseph, R. (2017). "Flexible regenerated cellulose/polypyrrole composite films with enhanced dielectric properties," Carbohyd. Polym. 157, 1024-1032. DOI: 10.1016/j.carbpol.2016.10.065

Sahu, B. B., Long, W., and Han, J. G. (2018). "Highly conductive flexible ultra thin ITO nanoclusters prepared by 3-D confined magnetron sputtering at a low temperature," Scripta Mater. 149, 98-102. DOI: 10.1016/j.scriptamat.2018.02.018

Sannicolo, T., Lagrange, M., Cabos, A., Celle, C., Simonato, J. P., and Bellet, D. (2016). "Metallic nanowire-based transparent electrodes for next generation flexible devices: A review," Small 12(44), 6052-6075. DOI: 10.1002/smll.201602581

Tang, Y., He, Z., Mosseler, J. A., and Ni, Y. (2014). "Production of highly electroconductive cellulosic paper via surface coating of carbon nanotube/graphene oxide nanocomposites using nanocrystalline cellulose as a binder," Cellulose 21(6), 45694581. DOI: $10.1007 / \mathrm{s} 10570-014-0418-9$

Tran, D. P., Lu, H. I., and Lin, C. K. (2015). "Effects of cyclic deformation on conductive characteristics of indium tin oxide thin film on polyethylene terephthalate substrate," Surf. Coat. Tech. 283, 298-310. DOI: 10.1016/j.surfcoat.2015.11.007

Wu, J., Liu, Y., Islam, A., Zheng, Q., Li, J., Ji, W., Chen, L., and Ouyang, X. (2020). "From straw to device interface: Carboxymethyl-cellulose-based modified interlayer 
for enhanced power conversion efficiency of organic solar cells," Adv. Sci. 7, 1902269-1902275. DOI: 10.1002/advs.201902269

Wu, R. L., Wang, X. L., Li, F., Li, H. Z., and Wang, Y. Z. (2009). “Green composite films prepared from cellulose, starch and lignin in room-temperature ionic liquid," Bioresource Technol. 100(9), 2569-2574. DOI: 10.1016/j.biortech.2008.11.044

Yan, J., and Xu, R. (2015). "Reinforced conductive polyaniline-paper composites," BioResources 10(3), 4065-4076. DOI: 10.15376/biores.10.3.4065-4076

Zhang, C., Lin, X., Zhang, N., Lu, Y., Wu, Z., Liu, G., and Nie, S. (2019a). "Chemically functionalized cellulose nanofibrils-based gear-like triboelectric nanogenerator for energy harvesting and sensing," Nano Energy 66, 104126-104130. DOI: 10.1016/j.nanoen.2019.104126

Zhang, H., Dou, C., Pal, L., and Hubbe, M. A. (2019b). "Review of electrically conductive composites and films containing cellulosic fibers or nanocellulose," BioResources 14(3), 7494-7542. DOI: 10.15376/biores.14.3.7974-7542

Zhang, S., Niu, G., Yu, H., and Huang, J. (2019c). "Enhanced conductive fiber of multiwall-carbon-nanotubes templated carbonization of cellulose," BioResources 14(4), 8656-8663. DOI: 10.15376/biores.14.4.8656-8663

Zheng, X., Huang, F., Chen, L., Huang, L., Cao, S., and Ma, X. (2019). "Preparation of transparent film via cellulose regeneration: Correlations between ionic liquid and film properties," Carbohyd. Polym. 203, 214-218. DOI: 10.1016/j.carbpol.2018.09.060

Article submitted: January 27, 2020; Peer review completed: April 25, 2020; Revised version received and accepted: April 29, 2020; Published: May 5, 2020.

DOI: $10.15376 /$ biores.15.3.4699-4710 